\title{
Local density of states around single vortices and vortex pairs: effect of boundaries and hybridization of vortex core states
}

\author{
A. S. Mel'nikov, D. A. Ryzhov, M. A. Silaev ${ }^{1}$ \\ ${ }^{1}$ Institute for Physics of Microstructures, Russian Academy of Sciences, 603950 Nizhny Novgorod, GSP-105, Russia
}

(Dated: November 27, 2018)

\begin{abstract}
The profiles of local density of states (LDOS) around single vortices and vortex pairs in type-II superconductors are studied taking account of the interference of quasiparticle waves experiencing Andreev reflection within the vortex cores, hybridization of vortex core states and normal reflection at the boundaries or defects. For subgap energy levels these interference effects reveal themselves in a nontrivial dependence of the positions of the LDOS peaks on the intervortex distance and sample size: the peak positions generally do not coincide with the superconducting phase singularity points. The LDOS profiles are calculated for three generic examples which can be realized, e.g., in mesoscopic superconductors: (i) vortex-vortex pair; (ii) vortex positioned near a flat boundary; (iii) vortex positioned in the center of a superconducting disk. The resulting evolution of the Andreev interference patterns could be observable by scanning tunneling spectroscopy techniques in mesoscopic superconductors or disordered vortex arrays.

PACS numbers: 74.25.Jb, 74.25.Qp, 74.78.Na
\end{abstract}

\section{INTRODUCTION}

Physics of vortex matter in superconductors has been a long standing topic of intensive research for many years. Over a last decade growing interest to this topic has been stimulated by technological advances allowing deeper insight into the structure of vortices as well as investigation of new exotic vortex states. In particular, local density of states (LDOS) measurements with the help of scanning tunneling spectroscopy techniques have been proven to be an effective tool of experimental study of electronic structure of Abrikosov vortices, $1,2,3,4,5$. The observation of the zero-bias anomaly of tunneling conductance at the center of each vortex in these experiments clearly confirmed the existence of bound vortex core states predicted by Caroli, de Gennes and Matricon $(\mathrm{CdGM})^{6}$. The wave functions of the subgap states are localized inside the vortex core because of the Andreev reflection of quasiparticles at the core boundary. For each individual vortex the energy $\varepsilon(\mu)$ of a subgap state varies from $-\Delta_{0}$ to $+\Delta_{0}$ as one changes the angular momentum $\mu$ defined with respect to the vortex axis. At small energies $|\varepsilon| \ll \Delta_{0}$ the spectrum is a linear function of $\mu: \varepsilon(\mu) \simeq-\mu \omega$, where $\omega \approx \Delta_{0} /\left(k_{F} \xi\right), \Delta_{0}$ is the superconducting gap value far from the vortex axis, $k_{F}$ is the Fermi momentum, $\xi=\hbar v_{F} / \Delta_{0}$ is the coherence length, $v_{F}$ is the Fermi velocity, and $\mu$ is half an odd integer. Note that hereafter we assume the Fermi surface to be a cylinder and neglect the dependence of the quasiparticle energy on the momentum component along the vortex axis $z$ considering a motion of quasiparticles only in $x y$ plane. Introducing a cylindrical coordinate system $(r, \theta, z)$ and defining an impact parameter as $b=-\mu / k_{F}=\left[\mathbf{r}, \mathbf{k}_{F}\right] \cdot \mathbf{z}_{0} / k_{F}$ the quasiclassical LDOS inside the isolated vortex core $(r \ll \xi)$ can be found as follows: $N=\left(k_{F} / 2 \pi \xi\right) \int_{0}^{2 \pi} \delta\left(\varepsilon-\omega k_{F} r \sin \left(\theta-\theta_{p}\right)\right) d \theta_{p}$. Here we evaluate the LDOS summing up over the quasi- particle states at the trajectories characterized by the orientation angle $\theta_{p}$. This expression yields a singularity of zero energy LDOS at the vortex center 7.8 .9 : $N=1 /(2 \pi \omega r \xi) \approx N_{0} \xi / r$, where $N_{0}=(1 / 2 \pi) m / \hbar^{2}$ is a normal metal LDOS. Smearing of energy levels due to scattering effects leads to a reduction of LDOS peak amplitude at the vortex center. However, the peak in the LDOS distribution survives even in "dirty" limit when a mean free path is smaller than a coherence length $l<\xi$ (see Refs. 10.11).

The increase in the external magnetic field results in the decrease in the intervortex distance which should be accompanied by the overlapping of wave functions describing the quasiparticle states bound to the neighboring vortex cores. This hybridization of vortex core states can perturb the CdGM spectra of isolated vortices and the circular symmetry of the LDOS peaks (see, e.g., Ref. 9). Note that the LDOS peak anisotropy may also reflect possible superconducting gap and Fermi surface anisotropy, and formation of the charge density wave order $\frac{1.4 .9}{.}$.

The goal of the present manuscript is to show by the example of mesoscopic superconductor that the interference of quasiparticle waves trapped in neighboring vortex cores can result even in more dramatic consequences for the LDOS profiles around the vortex configurations. These consequences are not limited just to the distortion of the LDOS peaks at individual vortex positions caused by the concrete symmetry of the system and/or vortex configuration. The peak positions themselves appear to be shifted from the superconducting phase singularity points which are usually considered as vortex centers. Changing the external magnetic field we can control the vortex configuration in the sample and switch between different Andreev interference patterns in LDOS. The distinctive features of the electronic structure of vortices in mesoscopic samples with sizes of several coherence lengths are controlled by two key factors: (i) quasi- 
particle tunneling between the neighboring vortex cores which becomes extremely important when the individual vortices merge into the giant (multiquantum) vortex ${ }^{12}$ with a winding number larger than unity $M>1$; (ii) normal scattering of quasiparticles at the sample boundary.

The multiquantum vortex provides a simple illustration of the above statement regarding the difference in the positions of the LDOS peaks and phase singularity points. Indeed, the subgap spectrum of such vortex contains $M$ anomalous spectral branches (per spin projection) ${ }^{13}$. Each anomalous branch intersects the Fermi level and contributes to the low-energy DOS. At low energies the spectrum has the following form $13,14,15,16$ :

$$
\varepsilon_{j}(\mu) \sim-\frac{\Delta_{0}}{k_{F} \xi}\left(\mu-\mu_{j}\right),
$$

where the index $j$ enumerates different spectral branches $(1<j<M),-k_{F} \xi \lesssim \mu_{j} \lesssim k_{F} \xi$. The LDOS profile corresponding to the spectrum (1) consists of a set of axially symmetric ring structures ${ }^{15,16}$. Note that for an even winding number the anomalous branch crossing the Fermi level at $\mu=0$ (i.e. at zero impact parameter) is absent and, as a result, the LDOS peak at the vortex center disappears. The splitting of a multiquantum vortex into the individual vortices should lead to the transformation of LDOS rings into a set of peaks positioned in the cores of individual vortices. The initial stage of this LDOS transformation for small intervortex distances was studied in Ref. 15 within a perturbation approach. A nonperturbative approach which allows to describe the spectrum transformation accompanying the decay of the multiquantum vortex has been suggested in Refs. ${ }^{17,18}$.

The second factor which is crucial in mesoscopic superconducting samples is a quasiparticle scattering at the sample boundaries which comes into play when vortices approach a superconductor surface or a size of superconducting sample is small enough. For vortices positioned rather close to the sample surface the effect of quasiparticle reflection at the boundary on the spectrum and total DOS was investigated recently in Refs. 18, 19,20. The early stage of transformation of the LDOS profiles for vortices approaching a flat boundary has been studied numerically in Ref. 21.

In the present paper we analyze the effect of both the hybridization of vortex core states and boundary scattering on the LDOS structures and for this purpose consider three generic examples: (i) vortex-vortex pair; (ii) vortex positioned near a flat boundary; (iii) vortex positioned in the center of a superconducting disk. We will show that the LDOS profiles can be obtained qualitatively from the spectra found in Refs. 17, 18, 19. Our qualitative considerations will be confirmed by the detailed numerical calculations.

This paper is organized as follows. In Sec. I we give an overview of the theoretical framework which is employed in this work, namely the Bogoliubov-de Gennes theory and the quasiclassical Eilenberger approach. We discuss the spectrum and LDOS patterns for multi vortex configurations in Sec. $11 \mathrm{~A}$ and address the case of a vortex in a small mesoscopic cylinder in Sec. IIIB. We give our conclusions in Sec. IV.

\section{MODEL AND BASIC EQUATIONS}

Our further consideration is based on two approaches: (i) Bogoliubov-de Gennes (BdG) equations; (ii) quasiclassical Eilenberger theory. The first one appears to be more transparent for the qualitative analysis of the interference effects and convenient for numerical calculation of LDOS profiles around the vortex placed in a superconducting disk. The Bogoliubov-de Gennes (BdG) equations for particle- $(u)$ and hole-like $(v)$ parts of the wave function have the following form:

$$
\begin{aligned}
-\frac{1}{2 m}\left(\hat{\mathbf{p}}-\frac{e}{c} \mathbf{A}\right)^{2} u+\Delta v & =\left(\varepsilon+\varepsilon_{F}\right) u, \\
\frac{1}{2 m}\left(\hat{\mathbf{p}}+\frac{e}{c} \mathbf{A}\right)^{2} v+\Delta^{*} u & =\left(\varepsilon-\varepsilon_{F}\right) v .
\end{aligned}
$$

Here $\Delta$ is a gap function, $\mathbf{A}$ is a vector potential, $\hat{\mathbf{p}}=$ $-i \hbar(\partial / \partial x, \partial / \partial y)$, and $\mathbf{r}=(x, y)$ is a radius vector in the plane perpendicular to the vortex axis. The LDOS can be expressed through the eigenfunctions of the $\mathrm{BdG}$ equation (2) in the following form (see, e.g., Ref 22):

$$
N(\mathbf{r}, \varepsilon)=\sum_{n}\left|u_{n}(\mathbf{r})\right|^{2} \delta\left(\varepsilon-\varepsilon_{n}\right),
$$

where $u_{n}(\mathbf{r})$ is electron component of quasiparticle eigenfunction corresponding to an energy level $\varepsilon_{n}$ (we sum over both positive and negative values $\varepsilon_{n}$ ). The eigenfunction has to be normalized:

$$
\int\left(\left|u_{n}(\mathbf{r})\right|^{2}+\left|v_{n}(\mathbf{r})\right|^{2}\right) d^{2} \mathbf{r}=1
$$

In general case BdG equations are rather complicated. A simplification can be obtained if one considers a quasiclassical approximation, assuming that the wavelength of quasiparticles is much smaller than the superconducting coherence length (see, e.g., Ref. 23). Within such an approximation, quasiparticles move along linear trajectories, i.e. straight lines along the direction of the quasiparticle momentum $\mathbf{k}_{F}=k_{F}\left(\cos \theta_{p}, \sin \theta_{p}\right)$. Generally, the quasiclassical form of the wave function can be constructed as follows: $(u, v)=e^{i \mathbf{k}_{F} \mathbf{r}}(\tilde{U}, \tilde{V})$, where $(\tilde{U}(\mathbf{r}), \tilde{V}(\mathbf{r}))$ is a slowly varying envelope function. Then the system (2) is reduced to the quasiclassical Andreev equations:

$$
\begin{aligned}
& \frac{\hbar \mathbf{k}_{F}}{m}\left(-i \hbar \nabla+\frac{e}{c} \mathbf{A}\right) \tilde{U}+\Delta \tilde{V}=\varepsilon \tilde{U}, \\
& \frac{\hbar \mathbf{k}_{F}}{m}\left(i \hbar \nabla+\frac{e}{c} \mathbf{A}\right) \tilde{V}+\Delta^{*} \tilde{U}=\varepsilon \tilde{V},
\end{aligned}
$$


which are defined at the linear trajectories determined by the direction of the quasiparticle momentum $\mathbf{k}_{F}$.

The quasiclassical approximation allows to develop a powerful method for calculation of the LDOS based on the solution of Eilenberger equations for quasiclassical propagator along the trajectories 24 . For numerical treatment of these equations we follow the Refs. 8,25 and introduce a Ricatti parametrization for the Green function. The essence of this method is a mathematical trick which allows to solve two first order Ricatti equations instead of fourth-order system of Eilenberger equations. Following Refs. 8, 21, 25 one can obtain:

$$
\begin{aligned}
& \hbar v_{F} \frac{\partial}{\partial x^{\prime}} a\left(x^{\prime}\right)+\left[2 \tilde{\omega}_{n}+\Delta^{*} a\left(x^{\prime}\right)\right] a\left(x^{\prime}\right)-\Delta=0, \\
& \hbar v_{F} \frac{\partial}{\partial x^{\prime}} b\left(x^{\prime}\right)-\left[2 \tilde{\omega}_{n}+\Delta b\left(x^{\prime}\right)\right] b\left(x^{\prime}\right)+\Delta^{*}=0,
\end{aligned}
$$

where $x^{\prime}=\left(\mathbf{k}_{F} \mathbf{r}\right) / k_{F}=r \cos \left(\theta_{p}-\theta\right)$ is the coordinate along trajectory, $\Delta(\mathbf{r})=|\Delta| e^{i \Phi}, \Phi(\mathbf{r})$ is a superconducting phase, $i \tilde{\omega}_{n}=i \omega_{n}+m \mathbf{v}_{F} \mathbf{v}_{s}$ is a Doppler-shifted Matsubara frequency, $\omega_{n}=\pi T(2 n+1)$ and

$$
\mathbf{v}_{s}=\frac{1}{2 m}\left(\hbar \nabla \Phi-\frac{2 e}{c} \mathbf{A}\right)
$$

is a gauge-invariant superfluid velocity. The LDOS may be expressed through the scalar coherence functions $a$ and $b$ as follows 21

$$
N(\mathbf{r}, \varepsilon)=\int_{0}^{2 \pi} \frac{d \theta}{2 \pi} \operatorname{Re}\left\{\frac{1-a b}{1+a b}\right\}_{i \omega_{n} \rightarrow \varepsilon+i \nu},
$$

where $\varepsilon$ is the quasiparticle energy measured from Fermi level and $\nu$ is a parameter which accounts for an elastic scattering. Throughout this paper we will assume the simplest model ${ }^{26}$ for the gap function distribution around an isolated vortex positioned at the origin $\Delta(\mathbf{r})=$ $\Delta_{0} f_{1}(\mathbf{r})$, where

$$
f_{1}(\mathbf{r})=\frac{x+i y}{\sqrt{x^{2}+y^{2}+\xi_{v}^{2}}}=\frac{r e^{i \theta}}{\sqrt{r^{2}+\xi_{v}^{2}}}
$$

with the core size $\xi_{v}=\xi$. To describe the system of two singly-quantized vortices positioned at $\mathbf{r}= \pm \mathbf{a} / 2=$ $\pm(a / 2,0)$ we fit the gap function as follows:

$$
\Delta(\mathbf{r})=\Delta_{0} f_{1}(\mathbf{r}-\mathbf{a} / 2) f_{1}(\mathbf{r}+\mathbf{a} / 2) .
$$

The image method allows us to reduce the problem of a vortex positioned at the point $\mathbf{r}=(a / 2,0)$ near a flat boundary $x=0$ to the one describing a vortex-antivortex pair with the antivortex situated at $\mathbf{r}=-(a / 2,0)$. For the latter case we choose the gap function in the form:

$$
\Delta(\mathbf{r})=\Delta_{0} f_{1}(\mathbf{r}-\mathbf{a} / 2) f_{1}^{*}(\mathbf{r}+\mathbf{a} / 2) .
$$

Certainly, the above simplest approximation for the gap function might not be enough to describe closely spaced vortex-vortex or vortex-antivortex pairs. However, the resulting LDOS patterns obtained below are mainly controlled only by the distance between the phase singularity points and appear to be weakly sensitive to the concrete profiles of the order parameter absolute value. The key features of the LDOS patterns appear to hold even in the limit of the zero $\xi_{v}$ value, i.e., even for phase vortices without any suppression of the gap value inside the cores.

Considering hereafter only the vortex configurations of the finite size comparable with several coherence lengths we neglect the vector potential which is known to give only a moderate renormalization of the interlevel spacing $\omega$ (see Ref. 27).

\section{SPECTRUM AND LOCAL DENSITY OF STATES}

\section{A. Vortex molecules}

We now proceed with the calculation of LDOS profiles for two systems: (i) vortex-vortex pair and (ii) vortex positioned near the flat superconducting boundary. The image method discussed in Ref. 18 allows us to show that the latter case is equivalent to the vortex-antivortex pair in the bulk provided we choose the distance between the vortex and antivortex to be two times larger than the distance from the vortex to the flat surface in the original problem. To elucidate our main results we start from a qualitative description of the quasiparticle spectra following Ref. 18 .

Let us consider two vortices oriented along the $z$ axis. In the plane $(x y)$ the corresponding phase singularity points (or vortex centers) are positioned at $\mathbf{r}_{1,2}=( \pm a / 2,0)$. For quasiparticles propagating along the classical trajectories parallel to $\mathbf{k}_{F}$ we introduce the angular momenta $\mu=\left[\mathbf{r}, \mathbf{k}_{F}\right] \cdot \mathbf{z}_{\mathbf{0}}=k_{F} r \sin \left(\theta_{p}-\theta\right)$ and $\tilde{\mu}_{i}=\mu-\left[\mathbf{r}_{i}, \mathbf{k}_{F}\right] \cdot \mathbf{z}_{0}$ defined with respect to the $z$-axis passing through the origin and with respect to the $i$-th vortex axis passing through the point $\mathbf{r}_{i}$, correspondingly. Neglecting the quasiparticle tunneling between the vortex cores the wave function can be found as a superposition of two states localized at different vortices and having close energies: $\varepsilon_{v 1}=-\omega\left[\mu-\left(k_{F} a / 2\right) \sin \theta_{p}\right]$ and $\varepsilon_{v 2}=-\omega\left[\mu+\left(k_{F} a / 2\right) \sin \theta_{p}\right]$. The transformation of the quasiclassical spectrum occurs due to the overlapping of the corresponding wave functions and can be analysed using a standard quantum mechanical approach describing a two-level system 28 , which yields the secular equation

$$
\left(\varepsilon-\varepsilon_{v 1}\right)\left(\varepsilon-\varepsilon_{v 2}\right)=(\delta \varepsilon)^{2},
$$

and the resulting splitting of isoenergetic lines near the degeneracy point in the $\mu-\theta$ plane $\left(\theta_{p}=0\right.$ for our case)

$$
\omega \mu=-\varepsilon \pm \sqrt{\omega^{2}\left(k_{F} a / 2\right)^{2} \theta_{p}^{2}+(\delta \varepsilon)^{2}} .
$$

The tunneling of quasiparticles between the vortex cores is determined by the exponentially small overlapping of 
wave functions localized near the cores and results in the splitting of the energy levels: $\delta \varepsilon \sim \Delta_{0} \exp (-a / \xi)$. Thus, the estimate for the splitting $\delta \mu \simeq \delta \varepsilon / \omega$ of isoenergetic lines in the $\left(\mu, \theta_{p}\right)$-plane reads (see Eq. (9)):

$$
\delta \mu(a) \sim k_{F} \xi \exp \left(-\frac{a}{\xi}\right) .
$$

In the case of a vortex-antivortex pair the noninteracting states localized at different vortices have the energies $\varepsilon_{v}=-\omega\left[\mu+\left(k_{F} a / 2\right) \sin \theta_{p}\right]$ and $\varepsilon_{a v}=\omega[\mu-$ $\left.\left(k_{F} a / 2\right) \sin \theta_{p}\right]$. Taking into account the overlapping of the corresponding wave functions states we obtain the secular equation (8) with $\varepsilon_{v 1}=\varepsilon_{v}$ and $\varepsilon_{v 2}=\varepsilon_{a v}$. Therefore, the quasiclassical orbits near the degeneracy point $\theta_{p}=0$ are defined by the following equation:

$$
\varepsilon= \pm \sqrt{(\omega \mu)^{2}+(\delta \varepsilon)^{2}}-\omega\left(k_{F} a / 2\right) \theta_{p} .
$$

The classically forbidden angular domain at $\varepsilon=0$ has the width $\delta \theta_{p}=4 \delta \varepsilon /\left(\omega k_{F} a\right)$. One can assume that the appearance of such classically forbidden domain explains the deep structure in the local DOS profile observed numerically in Ref. 21 for a vortex near the flat boundary of an $s$-wave superconductor. The classically forbidden angular domain results in the suppression of the overall $\operatorname{DOS}^{18}$ and we show below that this mechanism is responsible for the anomalous spectrum branch disappearance when the vortex exits the sample.

To study the transformation of spectrum in the entire range of intervortex distances we solve numerically the quasiclassical Andreev equations (44) to find isoenergetic lines in $\mu-\theta_{p}$ plane. The resulting quasiclassical orbits for a vortex-vortex pair with the intervortex distances $a=2.5 \xi$ and $a=1.5 \xi$ are shown in Fig. 17. The dotted lines show the orbits for $a=0$, i.e., for a doubly quantized vortex. The splitting of the quasiclassical orbits grows with the decrease in the size of the vortex molecule both for the two-vortex and vortex-antivortex configurations. When the intervortex distance is of the order $\xi$ the energy splitting becomes so large $\left(\delta \varepsilon \sim \Delta_{0}\right)$ that the simplified equations (9), (11) are no longer valid and the spectrum is disturbed in the whole angular domain $0<\theta_{p}<2 \pi$. One can clearly observe that with the decrease in the intervortex distance the CdGM orbits $b= \pm(a / 2) \sin \theta_{p}$ corresponding to zero energy in isolated vortices transform into two lines $\mu= \pm \mu_{0}$ which correspond to the spectrum of a doubly quantized vortex. Comparing the isoenergetic lines with the unperturbed CdGM ones one can see that the zero energy quasiclassical trajectories do not pass through the vortex center. It means that the LDOS peaks are necessary shifted from the vortex centers as the intervortex distance becomes small enough. This phenomenon can be understood considering the following qualitative arguments. Indeed, when the intervortex distance is large $a \gg \xi$ the equilibrium size of the vortex pair is determined by the stability condition yielding the zero value of Lorentz force acting on each vortex: $\mathbf{F}_{L} \propto \phi_{0}\left[\mathbf{z}_{0}, \mathbf{v}_{s}\right]=0$, where $\phi_{0}$ is a magnetic
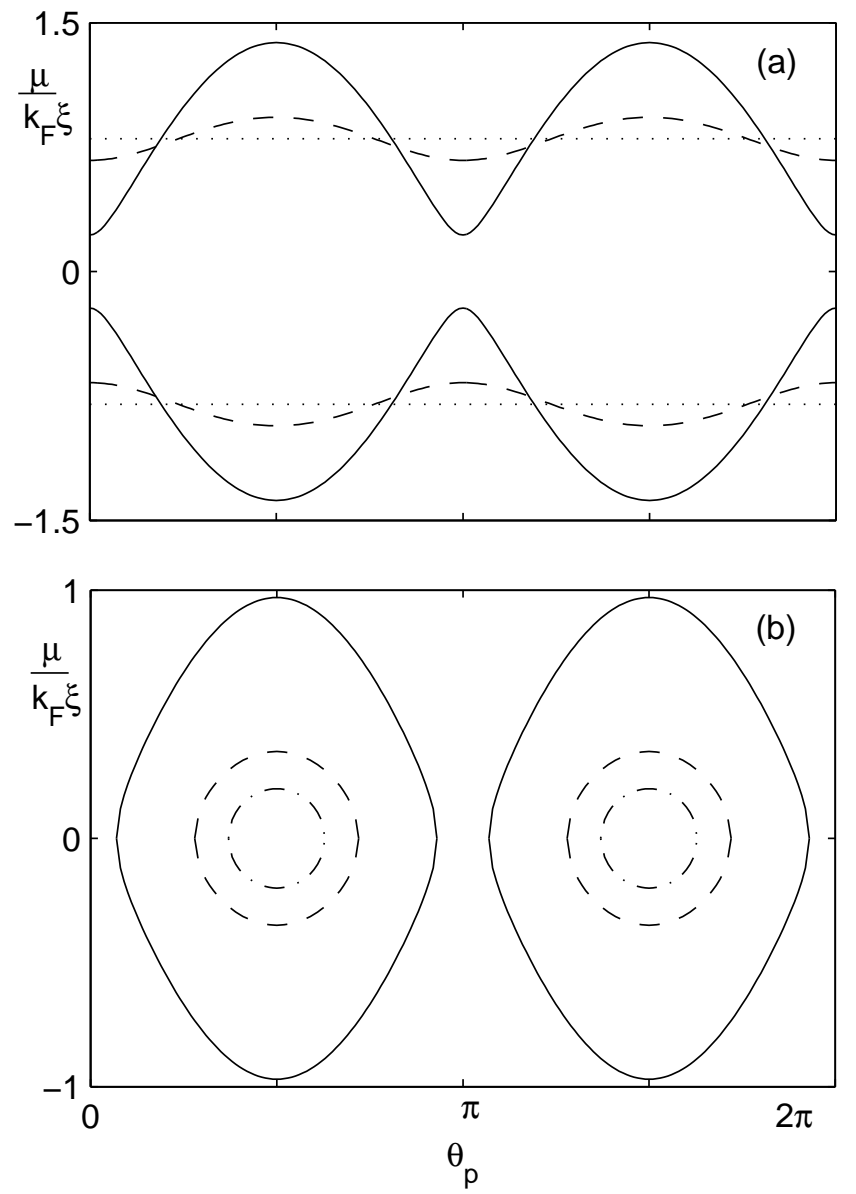

FIG. 1: Quasiclassical orbits in the $\mu-\theta_{p}$ plane corresponding to zero energy level. Upper panel (a): two vortices at distances $a=2.5 \xi$ (solid line) and $a=1.5 \xi$ (dashed line). Dotted lines correspond to the case of a doubly quantized vortex, i.e. to $a=0$. Lower panel (b): vortex-antivortex system with $a=2.5 \xi$ (solid line), $a=1.7 \xi$ (dashed line) and $a=1.6 \xi$ (dashdot line).

flux piercing the vortex and $\mathbf{v}_{s}$ is a a superfluid velocity induced in a given vortex core by other vortices and screening currents flowing along the boundaries. Thus, the local Doppler shift of the quasiparticle energy levels $\varepsilon_{d}=\hbar \mathbf{k}_{F} \mathbf{v}_{s}$ appears to vanish for trajectories passing through the vortex center. As a result, the position of the LDOS peaks coincide with the phase singularity points which are usually defined as vortex centers. However when the intervortex distance is comparable with (or less than) $\xi$ the part of the superfluid velocity induced by the neighboring vortex becomes strongly inhomogeneous inside the core region and the $\mathbf{v}_{s}$ value at a given vortex center diverges as $v_{s} \sim 1 / a$. As a consequence, the equilibrium size of the pair is no more determined by the condition of zero $\mathbf{v}_{s}$ at the vortex centers. The resulting nonvanishing Doppler shift for the trajectories passing through the vortex centers suppresses the LDOS at these points. Thus, for small $a$ the LDOS peak positions do 
not coincide with the vortex centers.

The Fig. 10 corresponds to the vortex-antivortex pair with the intervortex distances $a=2.5 \xi, a=1.7 \xi$, and $a=1.6 \xi$. One can see that contrary to the case of the vortex-vortex pair the decrease in the intervortex distance in this system leads to a rapid shrinking of the isoenergetic orbits. When the distance $a$ is small enough all the trajectories corresponding to the zero energy are characterized by the $\mu$ and $\theta_{p}$ values close to $\mu=0$ and $\theta_{p}=\pi(n+1 / 2)$, where $n$ is integer. For the intervortex distance $a / \xi=1.5$ the quasiclassical orbits completely shrink to these points in $\mu-\theta_{p}$ plane. In the real space the corresponding trajectories pass through the point in the middle between the vortex and antivortex and are perpendicular to the line connecting the phase singularity points. Thus, the two peaks of LDOS should shift from the vortex centers to the middle point and merge into one peak as the vortex and antivortex approach each other.

For a detailed study of these effects we calculate the LDOS profiles for two-vortex and vortex-antivortex configurations by solving numerically the Eilengerger equations (5) and applying the expression for the LDOS (6). The results are shown in Fig. 2 for the vortex-vortex pair with $a=1.5 \xi$ (upper panel) and $a=\xi$ (lower panel) and in Fig. 3 for a vortex-antivortex pair with $a / \xi=2$ (upper panel) and $a=1.5 \xi$ (lower panel). In Fig. 2 it is shown how the LDOS profile gradually transforms from a two-peaked structure corresponding to two isolated vortices (upper panel) to the axially symmetric ring for a doubly quantized vortex. The positions of the phase singularity points are shown on the contour plot by red crosses. It can be seen that even when the distance between the vortices exceeds the size of the vortex core the positions of the LDOS peaks do not coincide with the vortex centers. The distance between the LDOS peaks reduces slower than the size $a$ of the vortex molecule and finally the peaks are smeared into the anisotropic ring structure (see Fig. 2b). The anisotropy vanishes and the ring becomes axially symmetric when vortices merge into a doubly quantized vortex.

The evolution of the LDOS profile in a vortexantivortex pair follows a different scenario (see Fig. 33). When the distance between vortices is large enough there appear deep structures at the LDOS peaks (see Fig. 3a). The peaks themselves are slightly shifted towards the middle point at the line connecting the vortex and antivortex centers. Below the critical intervortex distance $a_{c} / \xi \sim 1.7$ the two peaks merge into one peak at the middle point (see Fig. 3b). As we discussed above this phenomenon is explained by the shrinkage of the quasiclassical orbits in the $\mu-\theta_{p}$ plane. When the vortex and antivortex come closer the amplitude of the LDOS peak is reduced and finally it disappears completely when the vortex and antivortex merge and annihilate. Mapping the solution of the vortex-antivortex problem on the one for a vortex near the flat surface we conclude that the zero bias LDOS peak appears to be positioned exactly at

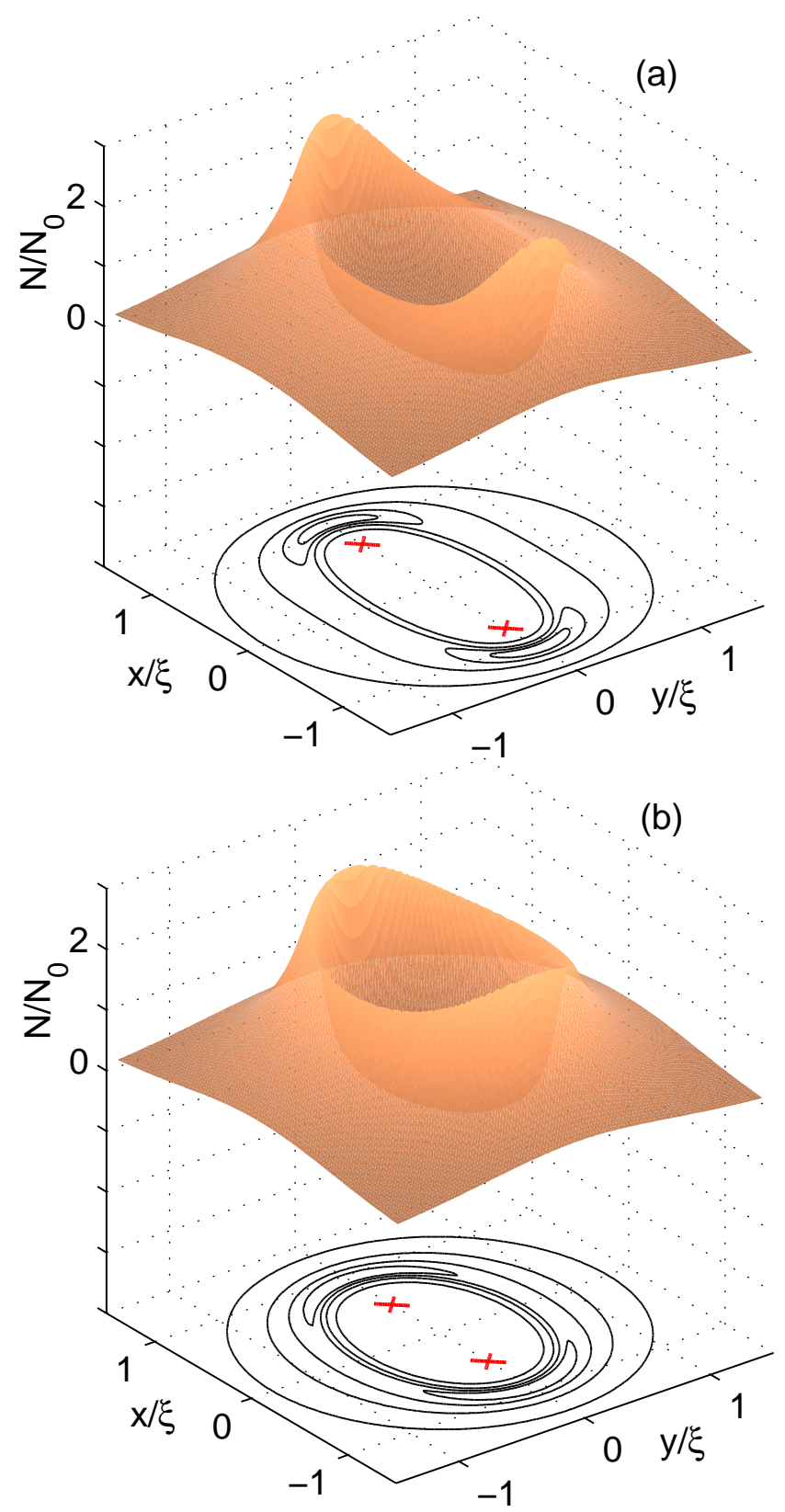

FIG. 2: (Color online) The normalized LDOS profile in a twovortex system for $a / \xi=1.5$ (a) and $a / \xi=1$ (b) calculated for $\nu=0.06 \Delta_{0}$. Positions of vortices are marked by red crosses.

the surface provided the distance from the vortex to the surface becomes less than $0.85 \xi$.

\section{B. Vortex in mesoscopic cylinder}

In this subsection we study another generic example illustrating the effect of the normal quasiparticle reflection at the sample boundary on the LDOS profiles in the vortex state. We consider a singly quantized vortex situated at the center of a superconducting disk of rather 

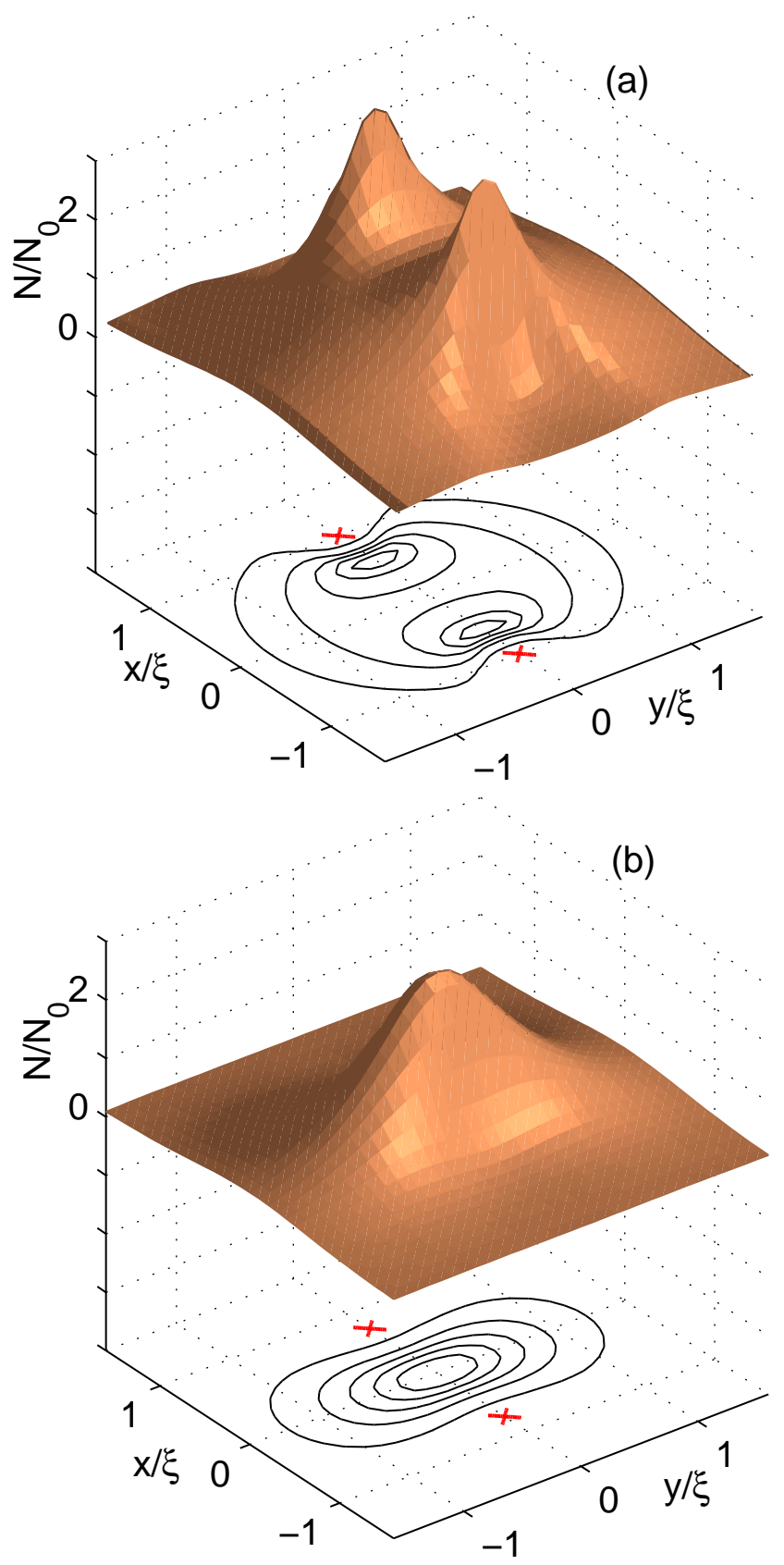

FIG. 3: (Color online) The normalized LDOS profile in a vortex-antivortex system for $a / \xi=2$ (a) and $a / \xi=1.5$ (b) calculated for $\nu=0.06 \Delta_{0}$. Positions of vortices are marked by red crosses.

small radius $R$ comparable with the coherence length. In this case a constructive interference of quasiparticle waves reflected from the boundary is known to result in mesoscopic oscillations of energy levels 19 :

$$
\varepsilon(\mu)=-\omega \mu-\delta \sin \left(2 k_{F} R-\pi \mu\right),
$$

where $\delta \sim \Delta_{0} \exp (-2 R / \xi)$ and $|\varepsilon(\mu)| \ll \Delta_{0}$. The remarkable fact is that at low energies these levels can be combined into two groups corresponding to odd and even values of the integer index $\mu+1 / 2$. A spacing between the energy levels belonging to one group is $2 \omega$ while the energy shift between the different groups is $2 \delta\left|\cos \left(2 k_{F} R\right)\right|$. Analogous shift of vortex core levels defined by the factor $\exp (-R / \xi)$ has been observed recently for a vortexantivortex pair on a sphere with a finite radius $R$ in $p_{x}+i p_{y}$ superconductors $\frac{29}{}$. Note that for a small superconducting disc with $R<R_{c} \sim(\xi / 2) \ln \left(k_{F} \xi\right)$ we have $\delta \gg \omega$. Therefore the two groups of energy levels can be considered as continuous branches if the energy discreteness with the scale $\omega$ is neglected. In this approximation one can use a quasiclassical expression $\mu=-k_{F} r \sin \left(\theta-\theta_{p}\right)$ and obtain the low energy branches in the form:

$$
\varepsilon_{1,2}=\omega k_{F} r \sin \left(\theta-\theta_{p}\right) \pm \delta \cos \left(2 k_{F} R\right) .
$$

Thus, for low energies $\varepsilon \ll \Delta_{0}$ the quasiclassical expression for the LDOS near the vortex center reads:

$$
N(r, \varepsilon)=\frac{k_{F}}{2 \pi \xi} \sum_{j=1,2} \int_{0}^{2 \pi} \delta\left(\varepsilon-\varepsilon_{j}\right) d \theta_{p} .
$$

The Eq. (14) can be derived from the general expression for LDOS (3) by setting the amplitude of the wave function $\left|\tilde{U}_{n}\right|^{2}=$ const which is a good approximation at the small distances $r \ll \xi$ from the vortex center. Evaluating this expression for the LDOS we obtain:

$$
\begin{array}{r}
N(r, \varepsilon=0)=N_{0} \frac{\xi}{\sqrt{r^{2}-r_{0}^{2}}}, \quad \text { for } r>r_{0}, \\
N(r, \varepsilon=0)=0, \quad \text { for } r<r_{0},
\end{array}
$$

where

$$
r_{0}=\frac{\delta\left|\cos \left(2 k_{F} R\right)\right|}{\omega k_{F}} \sim \xi\left|\cos \left(2 k_{F} R\right)\right| e^{-2 R / \xi} .
$$

Thus, the zero-bias peak of the LDOS at the vortex center which exists in an isolated vortex transforms into a ring structure which is similar to the LDOS pattern for a doubly quantized vortex. The only difference is that the radius of this ring is exponentially small comparing to the one in a doubly quantized vortex: $r_{0} / \xi \propto \exp (-R / \xi) \ll$ 1 . Therefore, this splitting of the LDOS peak in tunneling spectroscopy experiments should be strongly affected by different smearing effects like disorder scattering or finite temperature. In particular, for the observation of the above effect we should assume the elastic mean free path to exceed the characteristic size of the LDOS ring: $l \geq \xi \exp (-R / \xi)$. Note that this condition is not very restrictive because it can be fulfilled even in "dirty" superconductors with $l \ll \xi$ because typically $\exp (-R / \xi) \ll 1$. The effect of smearing due to the finite temperature is controlled by the parameter $T / \delta$.

To take into account the finite temperature effects and investigate the LDOS profiles in the whole range of energies and distances from the vortex core we solve numerically the BdG system (2) for a vortex in mesoscopic cylinder. We use the same method which was 
successfully applied to study the mesoscopic oscillations of vortex core levels $\frac{19}{9}$, the spectrum of multiquanta vortices in mesoscopic superconductors $\frac{18}{18}$ and the heat transport along vortex lines $\underline{20}$. The resulting spectrum as a function of the angular momentum is shown in Fig. 4 which clearly demonstrates the splitting of the anomalous CdGM branch at low energies caused by the boundary effects. For large energies $\varepsilon \sim \Delta_{0}$ the spectrum is

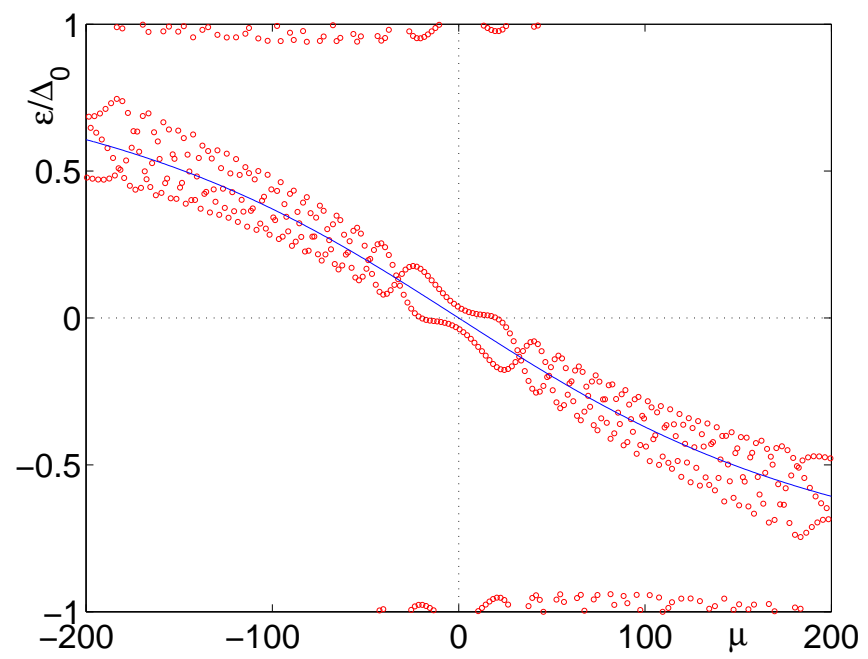

FIG. 4: (Color online) Quasiparticle spectrum vs $\mu$ for a singly quantized vortex in a disk of the radius $R=2 \xi$. The CdGM spectrum is shown by the solid line. We choose here $k_{F} \xi=200$.

not well described by the Eq.(12).

Using the spectrum and the expression (3) for the LDOS we can find the local differential conductance as a function of voltage $V$ :

$$
\frac{d I}{d V}=\left(\frac{d I}{d V}\right)_{N} \int_{-\infty}^{+\infty} \frac{N(\mathbf{r}, \varepsilon)}{N_{0}} \frac{\partial f(\varepsilon-e V)}{\partial V} d \varepsilon
$$

where $(d I / d V)_{N}$ is a conductance of the normal metal junction and $f(E)=[1+\exp (E / T)]^{-1}$ is a Fermi distribution function. The typical plots of the differential conductance are shown in Fig. 5 for different disk radii. The zero bias peak at the vortex center is clearly seen for a large disk radius when the normal scattering at the surface can be neglected. The decrease in the disk radius results in the shift of this conductance peak to higher voltages and formation of ring structure of local zero-bias conductance with finite radius $r \sim r_{0}$.

Note, that normal scattering of quasiparticles at the boundaries can be also important for multiquanta vortex configurations, e.g., vortex pairs, trapped in rather small samples. In our consideration of the LDOS profiles for a vortex-vortex pair in the previous subsection we have neglected the normal scattering at the sample surface assuming the vortex centers to be situated rather far from the boundaries. However, from our consideration of the vortex - antivortex problem one can expect that the

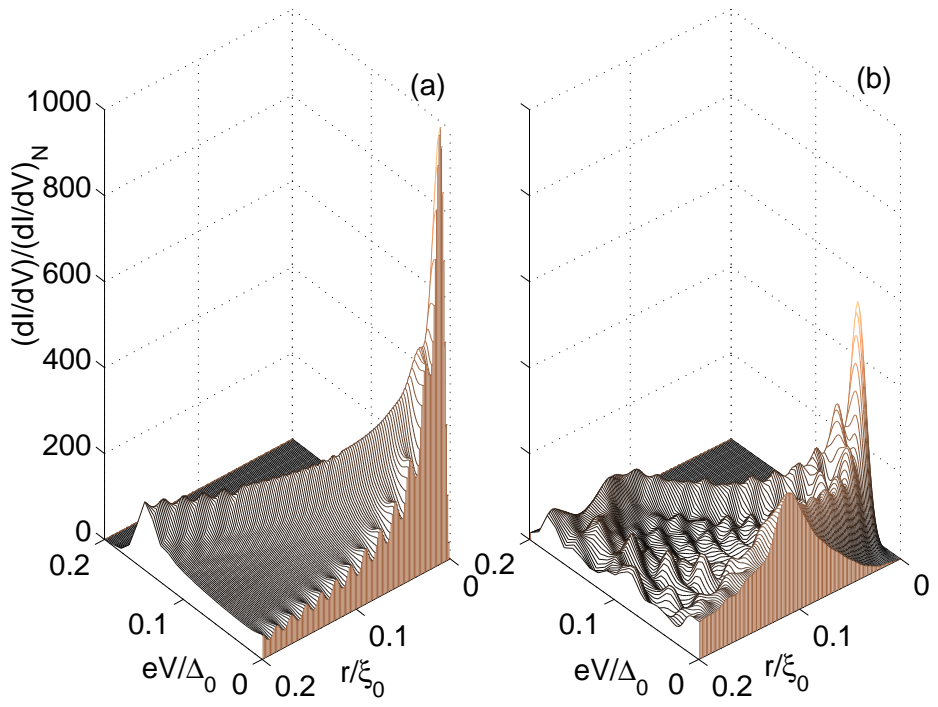

FIG. 5: (Color online) Three-dimensional plot of the normalized local differential conductance $d I / d V$ as a function of voltage $(e V)$ and distance from the vortex center $(r)$ in disks with a large radius $R=7 \xi$ (a) and small radius $R=2 \xi$ (b) for $T / \Delta_{0}=0.005$. We choose here $k_{F} \xi=200$.

effect of boundary scattering on the LDOS becomes important only when the distance from the vortex center to the sample edge is comparable with $\xi$. In this case the LDOS profiles in a vortex pair are affected by both the hybridization of the CdGM states and normal scattering. Considering the particular case of a doubly quantized vortex one can expect the boundary scattering to result in the spectrum oscillations (see Fig. 6) similar to the ones in a singly quantized vortex. These oscillations

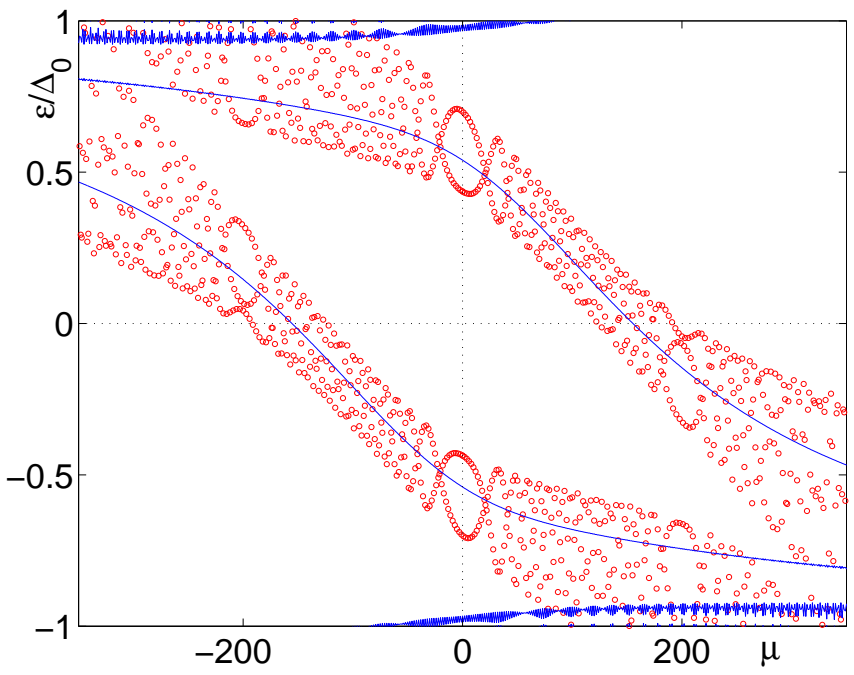

FIG. 6: (Color online) Quasiparticle spectrum vs $\mu$ for a doubly quantized vortex in a disk of the small radius $R=2 \xi$. The spectrum for a doubly quantized vortex in a disk of the large radius $R=7 \xi$ is shown by the solid line. We choose here $k_{F} \xi=200$. 
should cause the broadening of the ring in the LDOS pattern with the typical broadening scale $r_{0}$. The same effect should result in the broadening of the anisotropic ring structure for a vortex pair of a finite size.

\section{CONCLUSION}

To summarize, we have investigated the distinctive features of the LDOS profiles specific both for the vortex states in mesoscopic superconducting samples and for vortex arrays in bulk superconductors. These profiles are shown to be strongly affected by the Andreev interference effects and the normal scattering at the sample boundaries. In particular, we find that in contrast to the case of isolated vortices far from the boundaries the positions of peaks in the LDOS profiles in vortex configurations in small size samples are shifted from the phase singularity points. Taking a generic example of the two vortex system, we have considered the evolution of the LDOS profile which accompanies the merging of two vortices and appearance of the doubly quantized vortex. In this case the distance between LDOS peaks reduces slower than the intervortex distance $a$. Finally, when $a$ is smaller than the vortex core size the two-peaked structure of LDOS transforms into an anisotropic ring. The anisotropy vanishes when the vortex positions coincide and a doubly quantized vortex is formed.

Earlier (see Ref. 18) it was shown that the spectrum of a single vortex placed near the flat surface is transformed analogously to the vortex-antivortex system and when the distance is of the order of the vortex core size, the interlevel spacing in the vortex spectrum becomes larger than the CdGM value. This effect was argued to lead to the disappearance of the anomalous spectrum branch when the vortex approaches the surface. In the present paper we have confirmed this prediction by calculating LDOS profile for the entire range of distances from the vortex to surface. We have found an amazing effect for such system: as the vortex approaches the surface the zero-bias peak of LDOS shifts from the vortex center to the surface point positioned at the minimal distance to the vortex center. For the particular vortex core model that we have used in this paper the shift of the LDOS peak occurs when the dis- tance from vortex to surface is $0.85 \xi$. Experimentally the LDOS peaks positioned very close to the superconductor boundary have been recently observed in vortex state of tungsten (W) based thin films by scanning tunneling microscopy/spectroscopy techniques 30 . Note that in the latter experiment LDOS vortex peaks have been studied near the superconductor/normal metal $(\mathrm{Au})$ interface. We suppose that the normal scattering of quasiparticles at such boundary caused by either the Fermi velocity mismatch or some surface barrier can result in the transformation of the LDOS profiles analogous to the one discussed above for a superconductor/insulator boundary.

Also we have investigated a measurable consequence of the giant mesoscopic oscillations of vortex core levels in finite size superconducting systems $\frac{19}{}$. Considering the simplest case of a vortex positioned at the center of a superconducting cylinder with a radius $R$ we have shown that the zero-bias peak of the local differential conductance at the vortex center transforms into a ring structure of the radius of the order of $r_{0} \sim \xi e^{-2 R / \xi}$. We expect that the unusual behavior of the LDOS profiles which we have investigated could be observable in scanning tunneling microscopy/spectroscopy experiments in mesoscopic superconductors which are now in the focus of interest (see, e.g., Refs. 30,31,32).

Certainly, the Andreev interference patterns discussed above should be smeared by the disorder effects which are controlled by $\nu$ parameter within the Eilenberger theory. The resulting smearing of the LDOS profiles can be extremely important, e.g., for amorphous superconducting samples with rather small mean free path. Still we expect that the shift of the LDOS peaks from vortex singularity points caused by the Doppler effect will be observable even in this limit of strong disorder.

\section{ACKNOWLEDGEMENTS}

It is our pleasure to thank H. Suderow and N. B. Kopnin for stimulating discussions, and D. Roditchev for correspondence. This work was supported, in part, by Russian Foundation for Basic Research, by the Program "Quantum Physics of Condensed Matter" of RAS, and by the "Dynasty" Foundation.
${ }^{1}$ H. F. Hess, R. B. Robinson, R. C. Dynes, J. M. Valles, Jr., and J. V. Waszczak, Phys. Rev. Lett. 62, 214 (1989); H. F. Hess, R. B. Robinson, and J. V. Waszczak, Phys. Rev. Lett. 64, 2711 (1990).

2 B. W. Hoogenboom, M. Kugler, B. Revaz, I. MaggioAprile, O. Fischer, and Ch. Renner, Phys. Rev. B 62, 9179 (2000).

3 A. Kohen, Th. Proslier, T. Cren, Y. Noat, W. Sacks, H. Berger, and D. Roditchev, Phys. Rev. Lett. 97, 027001 (2006).
${ }^{4}$ I. Guillamon, H. Suderow, S. Vieira, L. Cario, P. Diener, and P. Rodiere, Phys. Rev. Lett. 101, 166407 (2008).

${ }^{5}$ O. Fischer, M. Kugler, I. Maggio-Aprile, C. Berthod, and C. Renner, Rev. Mod. Phys. 79, 353 (2007).

${ }^{6}$ C. Caroli, P. G. de Gennes, J. Matricon, Phys. Lett. 9, 307 (1964).

7 S. Ullah, A. T. Dorsey and L. J. Buchholtz, Phys. Rev. B 42, 9950 (1990).

8 N. Schopohl and K. Maki, Phys. Rev. B 52, 490 (1995).

9 N. Hayashi, M. Ichioka, and K. Machida, Phys. Rev. Lett. 
77, 4074 (1996); Phys. Rev. B 56, 9052 (1997).

10 A. A. Golubov and U. Hartmann, Phys. Rev. Lett. 72, 3602 (1994).

11 P. Miranovic, M. Ichioka, and K. Machida, Phys. Rev. B 70, 104510 (2004).

${ }^{12}$ G. Boato, G. Gallinaro, and C. Rizzuto, Solid State Commun. 3, 173 (1965); D. S. McLachlan, Solid State Commun. 8, 1589 (1970); V. A. Schweigert, F. M. Peeters, and P. S. Deo, Phys. Rev. Lett. 81, 2783 (1998); A. K. Geim, S. V. Dubonos, J. J. Palacios, I. V. Grigorieva, M. Henini, and J. J. Schermer, Phys. Rev. Lett. 85, 1528 (2000); L. F. Chibotaru, A. Ceulemans, V. Bruyndoncx, V. V. Moshchalkov, Nature (London) 408, 833 (2000).

13 G. E. Volovik, Pis'ma Zh. Eksp. Teor. Fiz. 57, 233 (1993) [JETP Lett. 57, 244 (1993)].

14 Y. Tanaka, A. Hasegawa, and H. Takayanagi, Solid State Commun. 85, 321 (1993); Y. Tanaka, S. Kashiwaya, and H. Takayanagi, Jpn. J. Appl. Phys. 34, 4566 (1995); D. Rainer, J. A. Sauls, and D. Waxman, Phys. Rev. B 54, 10094 (1996); S. M. M. Virtanen and M. M. Salomaa, Phys. Rev. B 60, 14581 (1999).

15 A. S. Mel'nikov and V. M. Vinokur, Nature, 415, 60 (2002); Phys. Rev. B 65, 224514 (2002).

16 K. Tanaka, I. Robel, and B. Janko, Proc. Natl. Acad. Sci. U.S.A. 99, 5233 (2002).

17 A. S. Melnikov and M. A. Silaev, Pisma Zh. Eksp. Teor. Fiz. 83, 675 (2006) [JETP Lett. 83, 578 (2006)].

18 A. S. Melnikov, D. A. Ryzhov, and M. A. Silaev Phys. Rev. B 78, 064513 (2008).

19 N. B. Kopnin, A. S. Mel'nikov, D. A. Ryzhov, I. A. Shereshevskii, V. I. Pozdnyakova, and V. M. Vinokur, Phys.
Rev. Lett. 95, 197002 (2005).

20 N. B. Kopnin, A. S. Mel'nikov, V. I. Pozdnyakova, D. A. Ryzhov, I. A. Shereshevskii, and V. M. Vinokur, Phys. Rev. B 75, 024514 (2007).

21 S. Graser, C. Iniotakis, T. Dahm, and N. Schopohl, Phys. Rev. Lett. 93, 247001 (2004).

22 J. D. Shore, Ming Huang, A. T. Dorsey, and J. P. Sethna, Phys. Rev. Lett. 62, 3089 (1989).

23 J. Bardeen, R. Kummel, A. E. Jacobs, and L. Tewordt, Phys. Rev. 187, 556 (1969).

24 G. Eilenberger, Z. Phys. 214, 195 (1968); A. I. Larkin and Y. N. Ovchinnikov, Zh. Eksp. Teor. Fiz., 55, 2262 (1968); [Sov. Phys. JETP, 28, 1200 (1969)]; J. W. Serene and D. Rainer, Phys. Rep. 101, 22 (1983).

25 N. Schopohl, arXiv:9804064.

26 J. R. Clem, J. Low Temp. Phys. 18, 427 (1975).

27 Brun E. Hansen, Phys. Lett. A 27, 576 (1968).

${ }^{28}$ L. D. Landau and E. M. Lifshitz Quantum mechanics. Nonrelativistic theory, (Pergamon, New York, 1991).

29 Y. E. Kraus, A. Auerbach, H. A. Fertig, and S. H. Simon, Phys. Rev. Lett. 101, 267002 (2008).

${ }^{30}$ I. Guillamon, H. Suderow, S. Vieira, A. FernandezPacheco, J. Sese, R. Cordoba, J. M. De Teresa, and M. R. Ibarra, Journal of Physics: Conference Series (to be published).

31 M. Zalalutdinov, H. Fujioka, Y. Hashimoto, S. Katsumoto, and Y. Iye, Physica B 284-288, 817 (2000).

32 T. Cren, D. Fokin, F. Debontridder, V. Dubost, and D. Roditchev, (unpublished). 